\title{
Optimal reactive power support for distributed Micro Grid through PV-Grid connected inverter
}

\author{
G. Vijai ${ }^{1}$ and P. Selvam ${ }^{2}$ \\ \{reachvijaigopal@gmail.com ${ }^{1}$, sumatselvam@gmail.com $\left.{ }^{2}\right\}$ \\ ${ }^{1} \mathrm{Ph} . \mathrm{D}$. Scholar, Department of Electrical and Electronics Engineering, Vinayaka \\ Mission's KirupanandaVariyar Engineering College, Vinayaka Mission's Research \\ Foundation (Deemed to be University), Salem, India \\ ${ }^{2}$ Professor, Department of Electrical and Electronics Engineering, Vinayaka Mission's \\ KirupanandaVariyar Engineering College, Vinayaka Mission's Research Foundation \\ (Deemed to be University), Salem, India
}

\begin{abstract}
Now a day, Distributed Generation in Micro Grids plays a major role in extracting energy at convenient places and distributing with much ease and efficiently. Under such circumstances maintaining the Voltage levels at the point of distribution or point of common coupling (PCC) is an important task as demanded by the loads. Hence balancing or sharing the real and reactive power among the connected inverters is a challenging task. Droop control strategies play a better role in sharing the power among the inverters, although in many circumstances maintaining the node voltages at the system network is an arduous task. In order to improve the voltage level at the distribution points/PCC of the inverters a simple technique is presented in this paper, which measures the deviation of the voltage level at PCC and feed accordingly the reactive power to boost the voltage. However, the real power sharing is undisturbed. The method is schemed and simulated using MATLAB Simulink environment. The simulated results are encouraging that the intended technique has very good efficacy in maintaining the voltage level and agreed with the expected results.
\end{abstract}

Keywords: Micro Grid, Grid connected Inverter, Distributed Generation, PR controller, PV Inverter, Parallel connected Inverters, Real Power flow, Reactive Power flow.

\section{Introduction}

Distributed generation in Micro Grids plays a significant role by the way of sharing real power and reactive power among various sources connected to improve system efficiency, stability, quality etc. Since the distributed generation on account of the use of renewable sources profusely forming decentralized Micro Grids providing good quality of power and reliability [1]. Generally, PV inverters which are connected to the grid operates as a current source, where the inverter works in synchronism with the grid, taking the grid reference with the help of PLL circuit. The inner current control loop ensures the inverter to work as a current source [2]. While transferring power to the grid, the quality of power transfer is an important factor thus ensuring minimal distortion thereby avoiding injection of harmonics. Under such cases current control inverter plays a crucial role on the quality of the current supplied to the grid by the PV inverter [3]. 
Voltage support to the grid is done by pumping reactive power to the grid. Many controls to pump real and reactive power to the grid is configured using droop control technique such that all the inverters share the power in proportion to their rating. Such control basically requires the inverters connected to the grid to work in voltage control mode. In many cases reactive sharing is influenced by the line impedance resulting errors in sharing reactive power among the inverters.

This paper concentrated in pumping all the real power as what obtained from the PV array to the grid, in addition to this, the inverter will pump appropriate magnitude of reactive power ensuring good voltage support at the point of common coupling. The inverter control proposed in this paper makes it to work as a gird feeding inverter, however the reactive power feed is controlled interactively upon the variation in the grid voltage at the point of common coupling. Hence sharing of reactive power is mandated by the change in the grid voltage at PCC. In another method [4], the reactive power sharing is made to the grid by the available current capability of the grid feeding inverter without delimiting the current capacity of the inverter.

Sharing of Real and Reactive power shall be implemented through many techniques, however in the case of grid connected PV inverters sourcing from PV sources, the prime objective will be transferring, the available irradiation dependent maximum possible power from the PV array to the grid. Hence under such circumstances conventional or any optimized sharing of powers shall not be possible. However, the PV grid connected inverters could support the grid voltage by pumping appropriate magnitude of reactive power. Under conventional control techniques for sharing power among the connected inverters in a micro grid, proper sharing of real and reactive power should be carried out, otherwise inverters with small capacities having reached their control limits, may have a chance to overload thereby tripping out of service [5][6]. In the case of control presented in this paper, the PV based grid connected inverter shares reactive power in correlation to the magnitude deviation of grid voltage from the nominal value without crossing the kVA capacity of the inverter, making the inverter to work safely.

\section{Structure of the proposed Grid connected Inverter}

The proposed structure of PV sourced grid connected inverter with LCL filter is shown in Fig. 1. The proposed structure consists of a string of Photovoltaic modules connected to a Boost converter which is controlled by a Maximum Power Point controller in order to extract maximum possible irradiation dependent power from the PV array. The output power from the Boost converter feeds the inverter through a DC link. The inverter is controlled in such a way that the power available at the DC link is completely processed and fed to the grid. This optimal power flow into the grid from the DC link is made by maintaining the DC link voltage at a pre-determined voltage level. A general-purpose Phase Locked Loop is used to synchronize the inverter with the grid and the power flow is managed with the help of a current controller. 


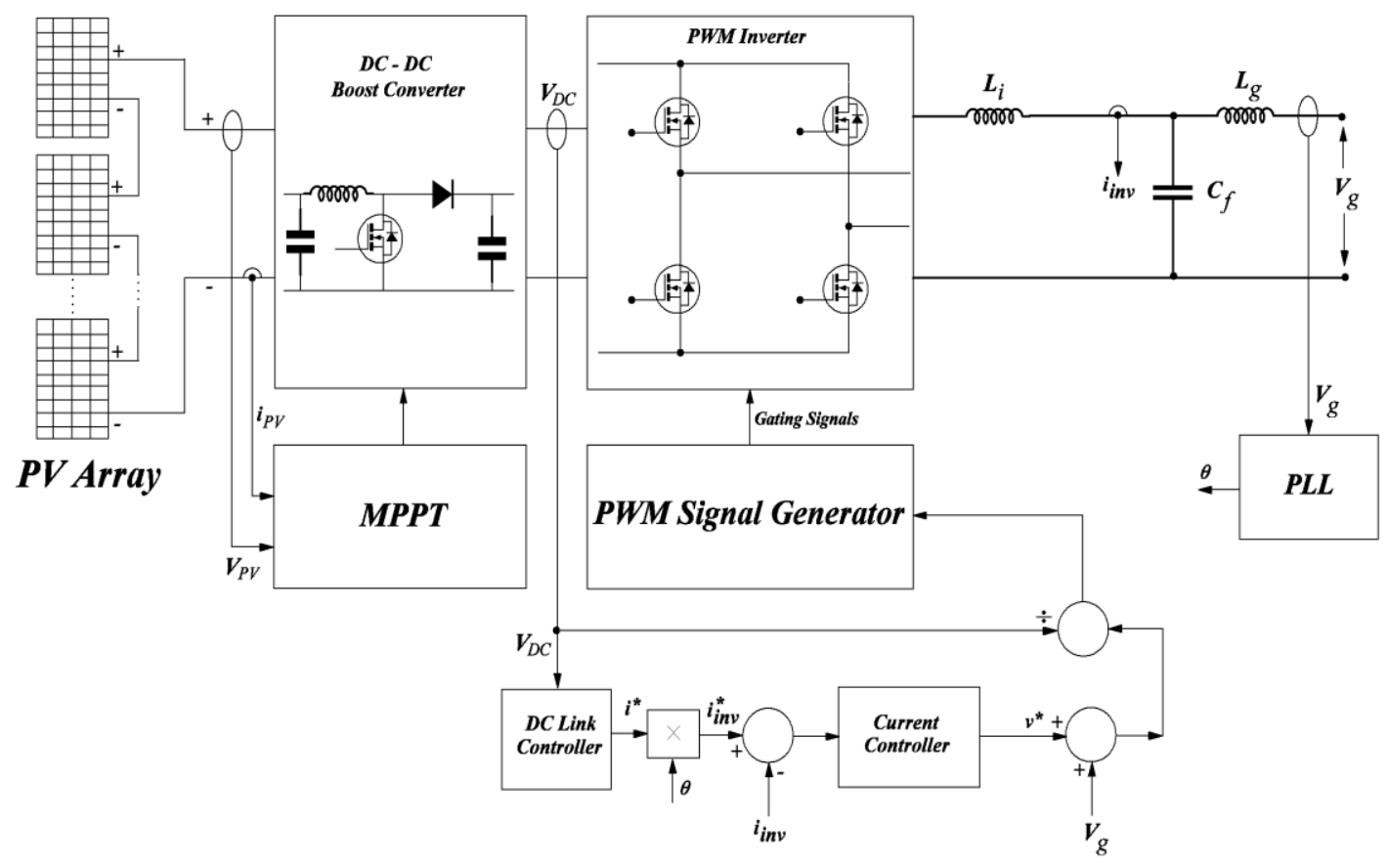

Fig 1.Structure of proposed Grid connected Inverter

The Maximum Power Point tracking algorithm used in this scheme is Perturb and Observe method with uniform level of irradiation. The MPP algorithm operates the Boost converter in such a manner that the PV array operates at the optimal operating point even when the level of irradiation changes. The DC link voltage controller is playing a crucial part so that the available power in the Dc link is completely transferred to the grid by maintaining a constant DC link voltage. The DC link voltage controller sets the real part power to be pumped into the grid.

The grid voltage is sensed and the deviation of the grid voltage from the desired magnitude is computed. Based on the magnitude of deviation, the reactive power to be pumped into the grid is determined. While determining the magnitude of reactive power, care is taken that under any circumstances the kVA rating of the inverter should never be exceeded. The real power from the current component estimate from the DC link voltage controller and the reactive power current component estimate from the reactive power estimation is combined with the help of PLL and a current reference is generated. This generated current reference makes the current controller to follow it such that the desired amount of real and reactive power flows into the grid.

\section{Real Power Support from the Inverter}

The real power support by the inverter depends solely on the level of irradiation on the PV array. As the irradiation changes time to time the real power support is also changing accordingly. From the inverter point of view the real power to be pumped into the grid is computed by maintaining the Dc link voltage which in turn depends on the Power fed in to the DC link by the Boost converter. 


\section{Reactive Power Support}

The reactive power estimation is done based on the grid voltage and the available volt-ampere capacity left over after deducting the real power current component. The grid voltage is sensed and the reactive power current component is estimated based on the equation (1) [7].

$$
i_{q}^{*}=\frac{\Delta V}{X_{g}}=\frac{V_{g}^{*}-V_{g}}{\omega L_{g}}
$$

If the estimated current is accommodated within the $\mathrm{kVA}$ capacity of the inverter, the net reference current is computed otherwise the estimated reactive power current component is scaled down such that the rated $\mathrm{kVA}$ of the inverter is not exceeded. The reactive power current component is estimated based on the expression presented in equation (2).

$$
i_{q}^{*} \leq \sqrt{i_{\text {rated }}^{2}-i_{p}^{* 2}}
$$

\section{Scheme of control of the proposed method}

The execution of the proposed scheme is based on estimation of the real power component of current and the reactive power component of current. The real power component of current is solely depending on the level of irradiation and is given by the DC link voltage controller. The reactive power component of current depends on two factors such as grid voltage magnitude and the inverter rating.

At every sampling the grid voltage is sampled and the reactive power component of current is estimated to bring the grid voltage to the rated magnitude and this estimated magnitude is then correlated with the rated current capacity of the inverter. If no violation is found the estimated value will be taken granted otherwise the estimation will be scaled such that the rating of the inverter should not be exceeded.

The scheme of control of the presented technique is illustrated in Fig. 2.

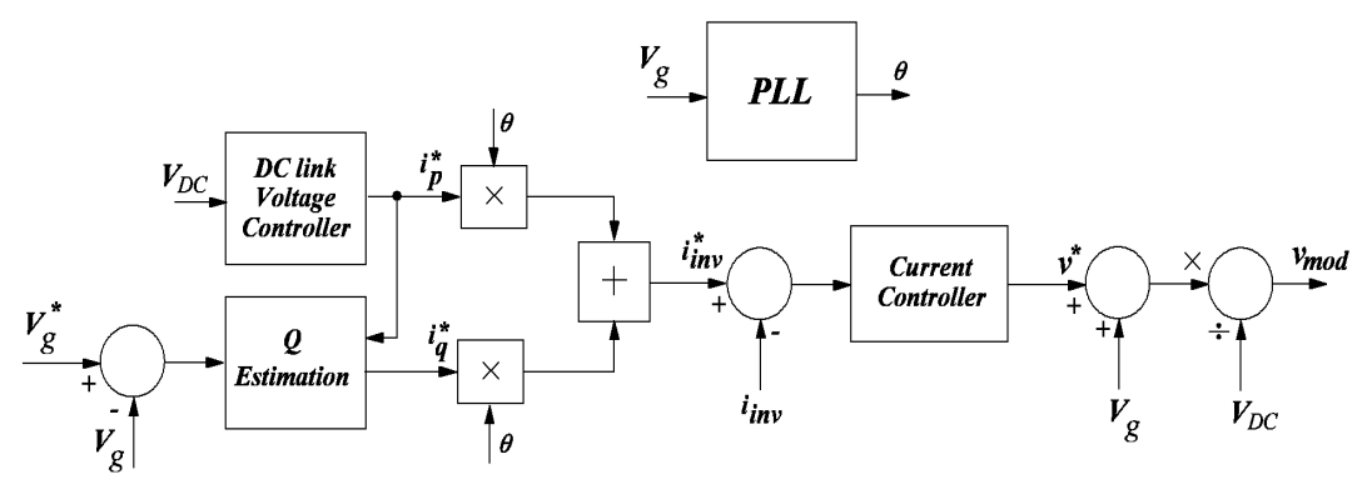

Fig 2. Control algorithm of the proposed technique

The obtained real and reactive component magnitudes of the reference current are combined with the help of the grid angle generated from the Phase Lock Loop. The reference 
current thus obtained is compared with the actual inverter current from the current sensor and the difference is passed to the Proportional and Resonant Controller [8], tuned to the grid frequency. The PR controller tracks the reference current in such a way that the desired real power and reactive power will flow to the grid.

As the reference current produced for the inverter is sinusoidal in nature, the tracking performance offered by the PR controller tuned at the grid frequency is excellent $[9,10]$.

\section{Synchronization}

In order to successfully inject the desired magnitude of real and reactive power to the grid, the reference current generated should be exactly matched with the grid frequency and phase. Hence the grid angle is a mandatory component to successfully generate the reference current from the computed real power component and the estimated reactive power component of current. The grid angle is extracted from the grid with the help of a generalpurpose Phase Locked Loop incorporating a moving average filter, digital PI controller and a digital integrator. The scheme of the PLL used is illustrated in the Fig. 3 as below.

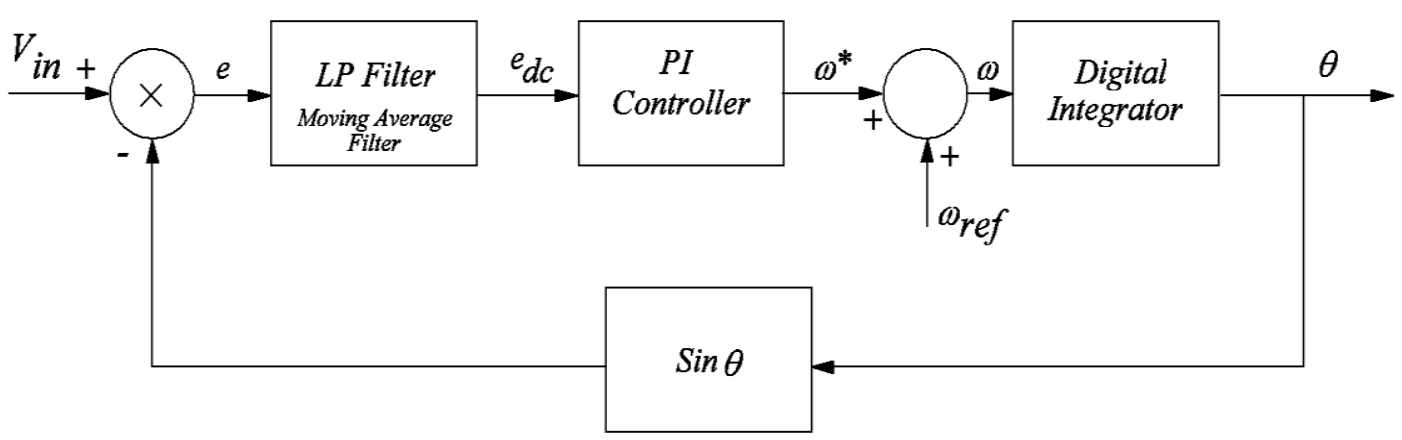

Fig 3. Digital Phase Locked Loop for Grid Synchronization

\section{Computer Simulation and Verification}

The methods behind proposed technique of optimal reactive power support are verified with the help of Simulink software from MATLAB ${ }^{\circledR}$. The scheme used to verify the proposed concept consists of two inverters connected in parallel, wherein one of the inverter supply energy from a battery source and acts as a grid forming inverter and the other inverter is fed from the PV source acts as grid feeding/supporting inverter [11]. The grid feeding inverter is connected with the grid through a digital phase locked loop and fed from a Boost converter, which acts as, in coordination with the controller, a Maximum Power Point Tracker. The power output from the Boost Converter is fed to the Dc link. The grid feeding/supporting inverter is operated in such a way that all the real power pumped into the DC link by the boost converter is in turn supplied to the grid, by maintaining a constant voltage, which is in this method is $400 \mathrm{~V}$ DC. The scheme of the proposed method contemplated in Simulink is illustrated in Figure $\mathrm{x}$. 


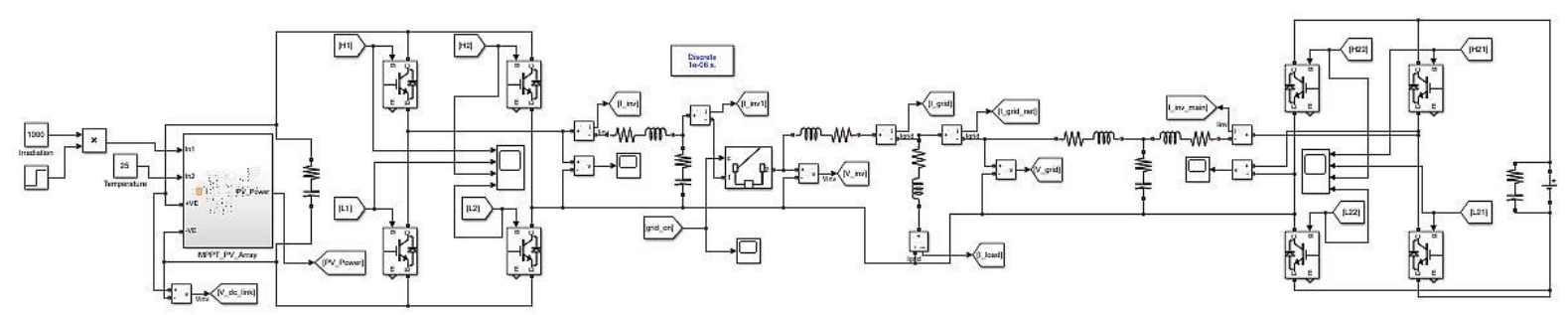

Fig 4. Simulation scheme for computer verification

The PV moduled used in the PV array for verification of the proposed technique is TSMC Solar TS-110C, which is in-built in Simulink package. In order to obtain the desired DC link voltage the number of $\mathrm{P}$ modules connected in series is found to be 10 . With the 10 numbers of PV modules in the array, the maximum power that can be delivered by the PV array at an irradiation of $1000 \mathrm{~W} / \mathrm{m}^{2}$, with a temperature at $25^{\circ} \mathrm{C}$ is $1098.71 \mathrm{Wp}$. The algorithm used to track maximum power point in the implementation is Perturb and Observe. The verification is done under uniform irradiation. The MPPT function is configured using MATLAB function.

The entire coding for the proposed method of optimal recative power support is implemented using S-function builder, which includes Digital PLL, proposed algorithm, PR controller. Discrete mode of simulation is used in the simulation model. With the help of Sfunctions, excellent performance is obtained in grid synchronization and the outcome of the proposed technique.

A common load of having real power and reactive power capacities $2000 \mathrm{~W}$ and 1500 Vars respectively is used in verifying the proposed technique through simulation. Two conditions of the grid voltage magnitude are used to verify the efficacy of the presented technique. In the first condition the grid voltage is set at a nominal magnitude of $230 \mathrm{~V} \mathrm{rms}$ and in the second condition the grid voltage magnitude is reduced to a value of $228 \mathrm{~V}$ rms to verify the reactive power contribution from the grid feeding/supporting inverter.

The response of the two inverters under the first condition (Grid Voltage $=230 \mathrm{~V}$ rms) is plotted in the figure illustrated in Figure 5 and Figure 6. It is clear that the real power supplied from the Grid is $1000 \mathrm{~W}$ and the real power pumped by the feeding/supporting inverter is also $1000 \mathrm{~W}$ as the PV array is set at $1000 \mathrm{~W} / \mathrm{m} 2$. However the reactive power support from the feeding/supporting inverter is 100Vars and the reaming 1400Vars are supplied by the Grid.

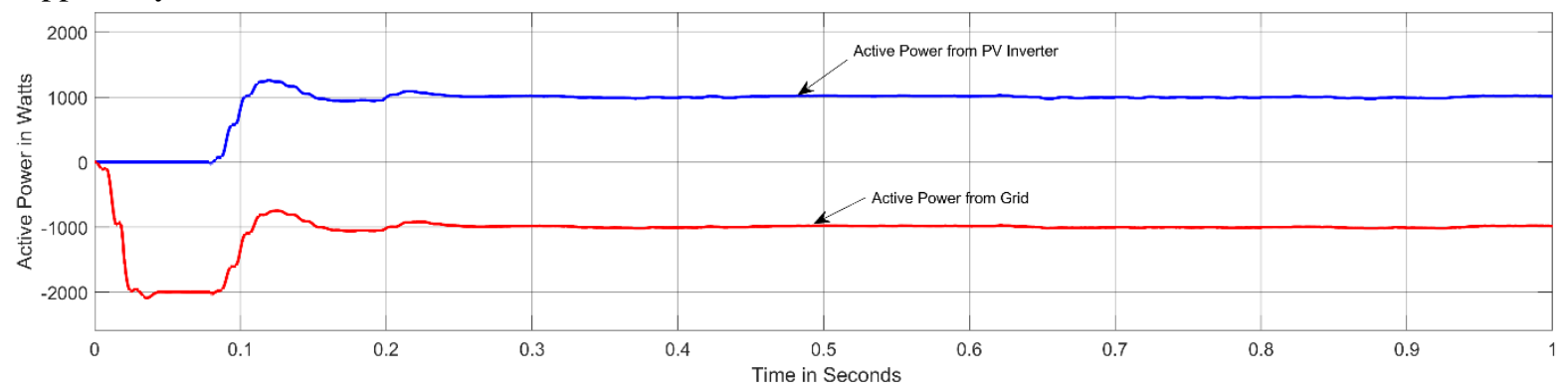

Fig 5. Real power sharing between the inverters ( $\mathrm{V}_{\text {grid }}$ is $\left.230 \mathrm{Vrms}\right)$ 


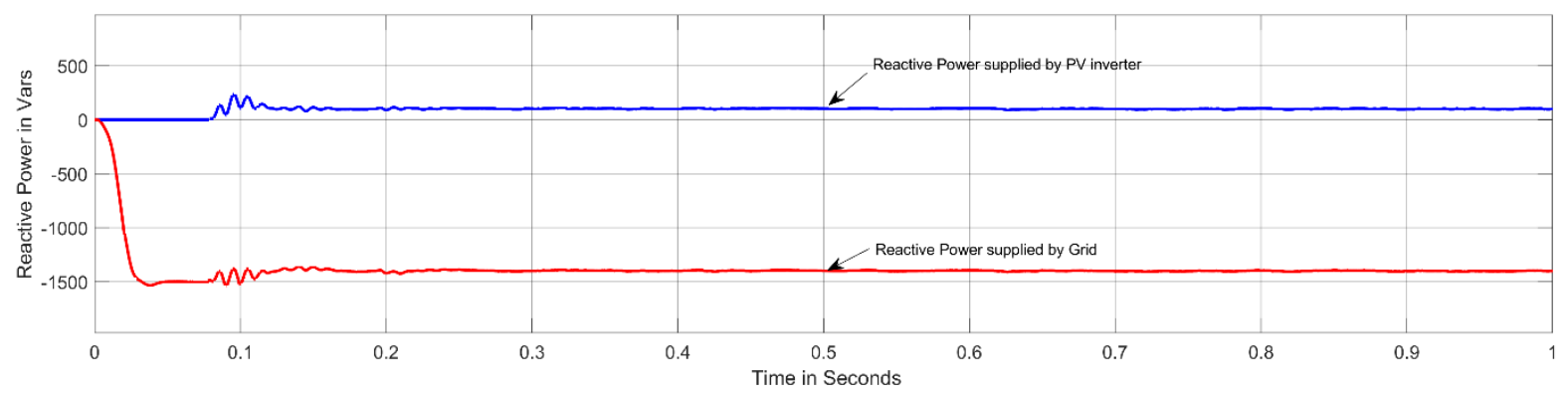

Fig 6. Reactive power sharing between the inverters ( $\mathrm{V}_{\text {grid }}$ is $\left.230 \mathrm{Vrms}\right)$

Under second condition (Grid Voltage $=228 \mathrm{~V} \mathrm{rms}$ ), the response is quite obvious that the algorithm works fine. The response of both the inverters under this condition is plotted in the figures illustrated in Figure 7 and Figure 8. In this case the algorithm senses that there is a decrease in the grid voltage and the difference is compensated by pumping sufficient reactive power by the feeding/supporting inverter in order to maintain the nominal magnitude of $230 \mathrm{~V} \mathrm{rms}$. The reactive power contribution from the feeding/supporting inverter to bring nominal grid voltage at the point of common coupling (PCC) is 540Vars. The remaining reactive power is shared by the Grid.

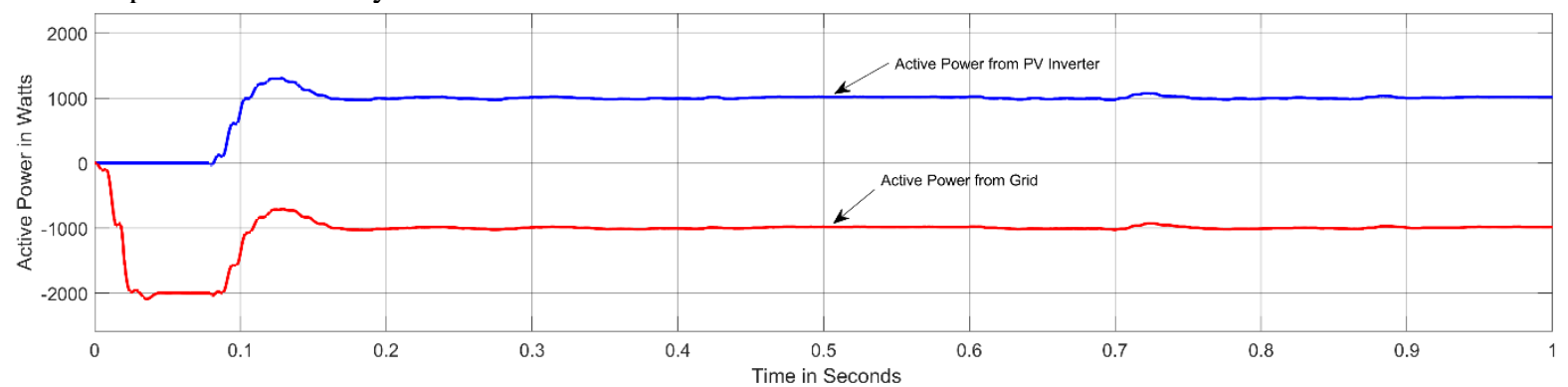

Fig 7. Real power sharing between the inverters ( $\mathrm{V}_{\text {grid }}$ is $228 \mathrm{Vrms}$ )

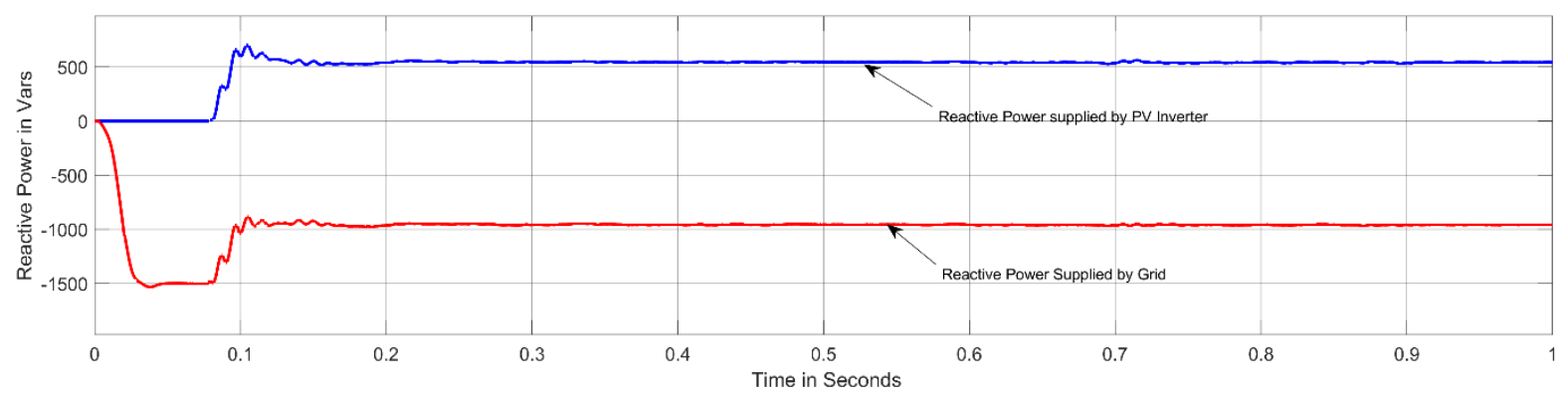

Fig 8. Reactive power sharing between the inverters ( $\mathrm{V}_{\text {grid }}$ is $228 \mathrm{Vrms}$ )

From the above discussions it is clear that the proposed algorithm works fine in compensating the voltage dips at the point of common coupling by injecting the required level of reactive power. Moreover, it also clear that the power pumped by the feeding/supporting 
inverter is constant irrespective of grid voltage and also there is no sensible change in the sharing of real power from the grid under small dip in the grid voltage.

\section{Conclusion}

An optimal reactive power support of grid feeding/supporting inverter is presented in this paper. The complexity of the so constructed technique is simple. The efficacy produced by the algorithm presented is effective in sharing of real and reactive powers as well as compensating the voltage dip at the point of common coupling. The reliability of the proposed technique is sufficiently good as it doesn't require communication techniques for sharing of powers. The simulation results produced through computer ratifies the efficiency and the efficacy of the proposed technique.

\section{References}

[1] Xuehua Wang et. al.,"Full Feedforward of Grid Voltage for Grid-Connected Inverter With LCL Filter to Suppress Current Distortion Due to Grid Voltage Harmonics", IEEE TRANSACTIONS ON POWER ELECTRONICS, VOL. 25, NO. 12, DECEMBER 2010

[2] Juan C. Vasquez et. al., "Voltage Support Provided by a Droop-Controlled Multifunctional Inverter", IEEE TRANSACTIONS ON INDUSTRIAL ELECTRONICS, VOL. 56, NO. 11, NOVEMBER 2009

[3] Daniel Zammit et. al., "Design of PR current control with selective harmonic compensators using Matlab", Journal of Electrical Systems and Information Technology 4 (2017) 347-358

[4] G. Vijai ,P. Selvam, "A Simple control of interactive voltage supporting PV-Grid connected inverter for distributed Micro Grid", Solid State Technology, Vol. 63 No. 62020.

[5] Yusuf Gupta et. al., "Optimal DG Allocation and Volt-var Dispatch for a Droop Based Microgrid", IEEE Transactions on Smart Grid, Volume: 12, Issue: 1, Jan. 2021, 169 - 181.

[6] Zhiyuan Tang et. al., "Distributed Coordinated Reactive Power Control for Voltage Regulation in Distribution Networks", IEEE Transactions on Smart Grid, Volume: 12, Issue: 1, Jan. 2021, pp 312 $-323$.

[7] Josep M. Guerrero et al., "Control of Distributed Uninterruptible Power Supply Systems", IEEE Transactions on Industrial Electronics, Vol. 55, No. 8, 2008.

[8] Tao Ye et. al., "Analysis, Design, and Implementation of a Quasi-Proportional-Resonant Controller for a Multifunctional Capacitive- Coupling Grid-Connected Inverter", IEEE Transactions on Industry Applications, Vol. 52, No. 5, Sep/Oct 2016.

[9] R. Teodorescu et al., "Proportional-resonant controllers and filters for grid-connected voltagesource converters", IEE Proc.-Electr. Power Appl., Vol. 153, No. 5, September 2006.

[10] JaumeMiret et al., "Selective Harmonic-Compensation Control for Single-Phase Active Power Filter With High Harmonic Rejection", IEEE Transactions on Industrial Electronics, Vol. 56, No. 8, 2009.

[11] J. Rocabert, A. Luna, F. Blaabjerg and P. Rodríguez, "Control of Power Converters in AC Microgrids," in IEEE Transactions on Power Electronics, vol. 27, no. 11, pp. 4734-4749, Nov. 2012 